\title{
IMPROVING DOPPLER FREQUENCY ESTIMATION FROM ENVISAT DATA
}

\author{
Thomas Kromer, Camilla Brekke \\ UiT - The Arctic University of Norway \\ Department of Physics and Technology \\ N-9037 Troms $\varnothing$
}

\author{
Harald Johnsen \\ Northern Research Institute \\ P.O. Box 6434 Forskningsparken \\ N-9294 Troms $\varnothing$
}

\begin{abstract}
This paper shows that Envisat ASAR data are degraded by a small periodic variation in gain between internal calibration cycles of the antenna, which introduces a significant bias when we try to estimate line-of-sight surface velocities from the estimated Doppler frequency shifts. We investigate the impact of the gain problems on the derived surface velocity product and propose a simple correction of the raw data.
\end{abstract}

Index Terms - Synthetic aperture radar, Doppler frequency estimation

\section{INTRODUCTION}

The azimuth center frequency or Doppler centroid is an important parameter when focusing synthetic aperture radar (SAR) images. Inaccurate estimation of this parameter leads to artifacts in the intensity image and will also affect other processing aspects such as geolocation accuracy [1].

There is a growing literature on estimation of the radial (across-track) surface velocities of scattering elements on the Earth surface with applications for ocean wind and surface current retrieval $[2,3]$. It has also been shown to be valuable for studying sea ice drift [4]. Our current work on estimating sea ice drift speeds from SAR Doppler estimates has revealed that Envisat Advanced Synthetic Aperture Radar (ASAR) data contain a periodic variation in gain which has a negative impact on the Doppler estimation process. Although the bias is small, it is still significant when we want to measure the Doppler centroid with high precision.

\section{BACKGROUND}

As a SAR satellite moves along its orbit (along track or azimuth direction) the antenna transmits microwave pulses in a side-looking geometry (across track or range direction) which are scattered by elements on the surface of the Earth. Some part of the incident radiation is reflected back towards the antenna and received by the SAR system. The SAR system records the Doppler shift history as it approaches and recedes from scatterers on the ground. The Doppler centroid $f_{\mathrm{Dc}}$ is a function of the relative motion between the satellite platform and a target on the surface of the rotating Earth

$$
f_{\mathrm{Dc}}=-\frac{2 v_{\text {rel }}}{\lambda}
$$

where $v_{\text {rel }}$ is the relative velocity between the SAR instrument and the Earth surface and $\lambda$ is the carrier wavelength of the SAR system [1]. The sign convention is such that the Doppler frequency is positive for scattering elements approaching the radar and negative for elements moving away from the radar.

Based on the geometry of the satellite orbit and attitude relative to the rotating Earth it is possible to predict the expected Doppler shift of a stationary scatterer on the Earth surface [1]. In general, Doppler shifts observed from the received signal do not agree perfectly with the predicted Doppler shifts. It has been shown that this anomaly contains a geophysical component related to the movement of scattering elements on the surface of the Earth $[2,3]$. The quantity of interest is therefore this residual motion.

We define the Doppler centroid anomaly as

$$
f_{\text {Dca }}=f_{\text {Dc }}-f_{\text {geom }}
$$

where $f_{\mathrm{Dc}}$ is the observed Doppler centroid defined as the radar return frequency shift at the antenna beam center and $f_{\text {geom }}$ is the Doppler shift predicted from satellite-Earth geometry [1].

The Doppler centroid is estimated from SLC data on a regular $1 \times 1 \mathrm{~km}^{2}$ grid with a resolution of $2 \times 2 \mathrm{~km}^{2}$ using the algorithm presented by Bamler (see [5]) modified to compensate for side band effects (see [6]). The output from the estimation process is a reduced resolution normalized cross section (NRCS) image, a Doppler centroid anomaly which can be converted to a radial velocity (RVL) image and the model Doppler frequency standard deviations (STD).

A geophysical Doppler shift relates to the Doppler centroid anomaly by

$$
f_{\text {phys }}=f_{\text {Dca }}-f_{\text {bias }}
$$

where $f_{\text {bias }}$ are the total errors due to uncertainties in the Doppler estimation, orbit, attitude, antenna mispointing and topography. 
We can then find the ground range line-of-sight surface velocity as:

$$
v_{\mathrm{rel}}=-\frac{\lambda f_{\mathrm{geo}}}{2 \sin \theta_{i}} .
$$

\section{ENVISAT ASAR GAIN VARIATION}

Figure 1 shows an example of the output from the Doppler estimation routine for a rainforest scene, where (a) shows the intensity, (b) shows the estimated geophysical Doppler $f_{\text {phys }}$ and (d) shows the model standard deviations. Note that each cell in the grid is $1 \times 1 \mathrm{~km}^{2}$ so the azimuth and range cell index corresponds approximately to distance in $\mathrm{km}$. A periodic variation similar to scalloping can be seen in the estimated geophysical Doppler grid (figure 1b). If we average the estimated Doppler frequencies over range we obtain the profile shown in figure 2 which reveals a sinusoidal pattern with an amplitude in the order of $2 \mathrm{~Hz}$ depending on the investigated scene. An error of $2 \mathrm{~Hz}$ corresponds to a $\sim 5.6 \mathrm{~cm} / \mathrm{s}$ error in the estimated radial velocities for the ASAR instrument which operates in the C-band.

This variation is clearly not geophysical in nature and is thus an additional bias that should be removed. Previously we have removed this bias as a post-processing step by estimating the parameters of a sinusoid with several harmonics from this profile to create a correction signal which is subtracted from each column of the Doppler grid. However, estimation of the amplitudes in non-homogeneous scenes could not be done reliably. The frequency of the pattern could be estimated reliably, however, and it was found that the signal has the same frequency as the internal calibration loop over the antenna elements. Closer inspection of the raw data revealed that the signal originates from a very small difference in gain between calibration cycles.

After the initial calibration within each scene, ASAR image mode raw data will have a gap in acquisition every 1023 lines to calibrate internal electronics. It is customary to replace the calibration line with a neighboring line of echo data. In the following description we will let gate denote a range of 1023 lines between these calibration lines. Figure 3 shows the average raw data intensity for each line across three gates, where the middle gate has a slightly higher gain than the two side gates. The problem at hand is thus to estimate the variation in gain between gates so that a correction of the raw data can be applied.

\section{BIAS CORRECTION}

From looking at several scenes it seems that a good approximation is that every other gate has a low/high gain compared to its neighbor and that the difference in gain between gates is reasonably stable within a scene. This observation and the fact that we know exactly where the change in gain occurs (at the start of a new gate) allows a very simple correction to the raw data that will remove the bias from the derived Doppler estimate.

Consider a set of three neighboring gates (see figure 3). Assume that there is a slowly varying curve underlying the profile which can be well-approximated by a polynomial of order $K=7$. The parameters $\left\{c_{s k}\right\}_{k=0}^{K}$ of this polynomial are then estimated using least squares fitting to the data from the side gates only (red part),

$$
I_{s}\left(t_{y}\right)=c_{s 0}+\sum_{k=1}^{K} c_{s k} t_{y}^{k}
$$

where $s$ refers to the side gates and $I\left(t_{y}\right)$ denotes intensity at relative azimuth time $t_{y}$ from the first pixel among the three gates. The estimated curve is shown covering all three gates in figure 3 . We then assume that the curve describing the middle gate, $I_{m}$, should have the same shape as $I_{s}$, but a different offset, i.e.

$$
I_{m}\left(t_{y}\right)=c_{m 0}+\sum_{k=1}^{K} c_{s k} t_{y}^{k} .
$$

We find $c_{m 0}$ by least squares fitting of (6) to the points in the profile corresponding to the middle gate only (green part). The resulting curve is shown covering only the middle gate in figure 3. From the two estimated curves we can calculate a gain correction factor $\delta$ from the ratio of the curves at the time $t_{c}$ corresponding to the first calibration line:

$$
\delta^{2}=\frac{I_{s}\left(t_{c}\right)}{I_{m}\left(t_{c}\right)}
$$

Typical values of $\delta^{2}$ were in the range $0.98-1.02$.

To obtain a good estimate we apply this procedure to every other gate in the image to obtain $N$ realizations of $\delta^{2}$. We then take as our estimated gain correction factor

$$
\hat{\delta}=\sqrt{\operatorname{median}\left(\left\{\delta_{n}^{2}\right\}_{n=1}^{N}\right)} .
$$

One could also obtain twice as many samples of $\delta^{2}$ by applying the procedure to every gate, to obtain $\delta_{1}^{2}, \delta_{2}^{-2}, \delta_{3}^{2}, \delta_{4}^{-2}$, etc. The raw data can now be adjusted by multiplying the raw data corresponding to every other gate by the estimated gain factor $\hat{\delta}$.

Note that the order $K$ of the polynomial was chosen somewhat arbitrarily to be flexible enough to model the azimuth profiles, yet stiff enough to prevent overfitting. The value has been effective for sea ice and ocean scenes, but it may be necessary to experiment with different values of $K$ for more complex scenes. Because the scenes used in this work were largely homogeneous, the routine may be improved by estimating the gain correction factor from homogeneous sub-images instead of averaging across all range pixels. 

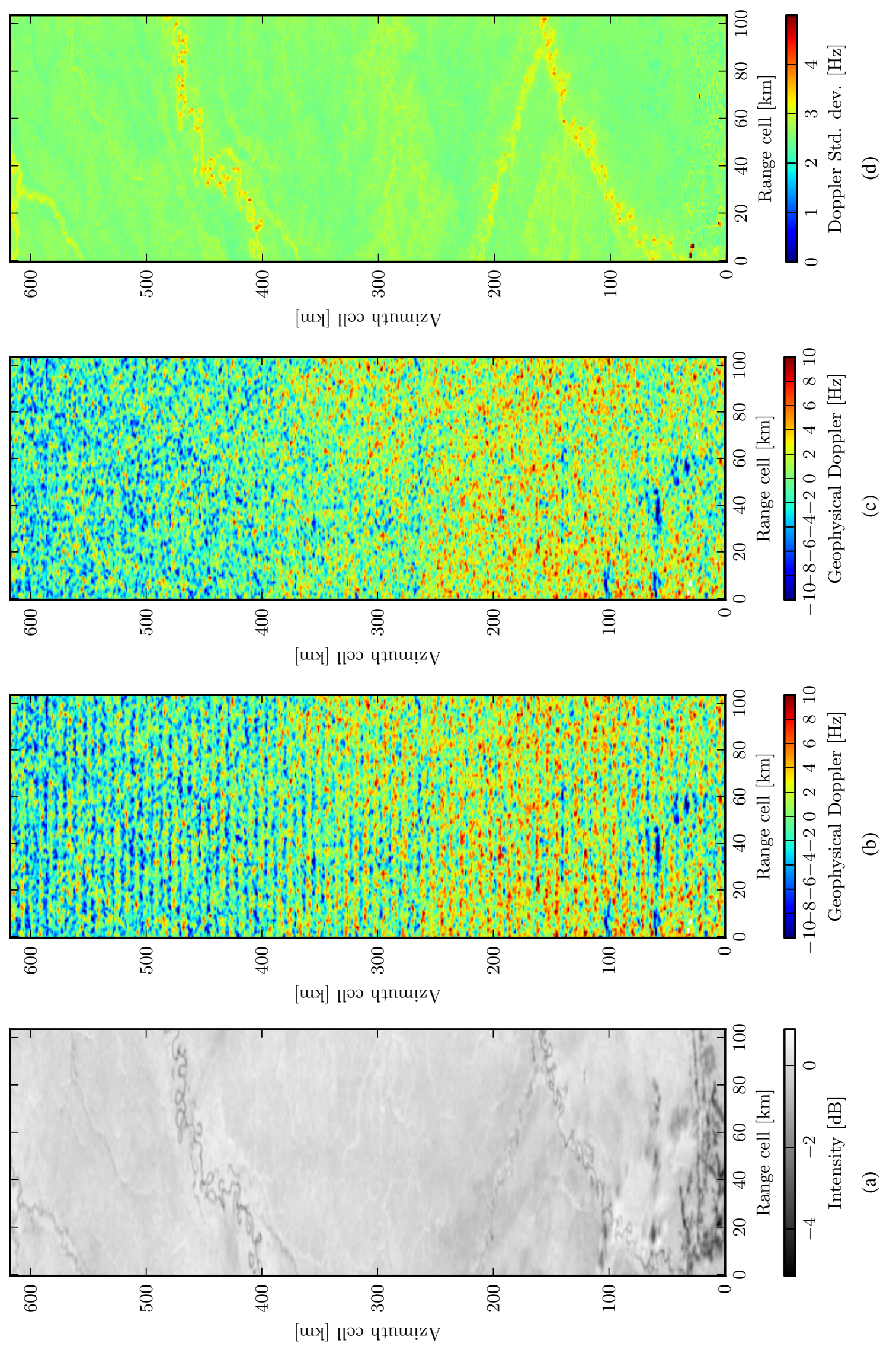

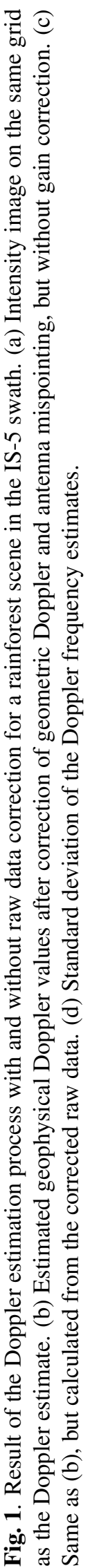




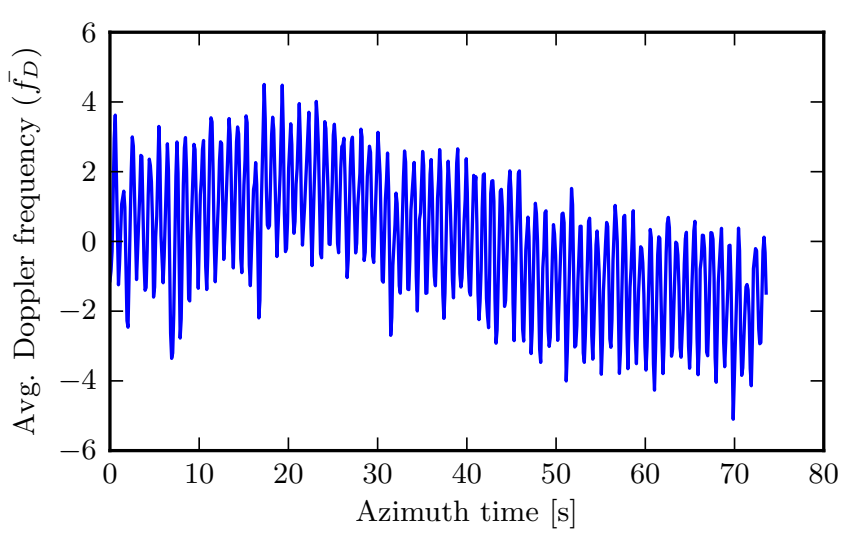

Fig. 2. Profile in the azimuth direction obtained by averaging the Doppler frequencies in figure $1 \mathrm{~b}$ along the range direction, showing a sinusoidal bias in the estimated Doppler frequencies.

\section{RESULTS}

The result of estimating the Doppler frequencies after applying the procedure described in section 4 is shown in figure $1 \mathrm{c}$. If we compare $1 \mathrm{c}$ to $1 \mathrm{~b}$, we see that the periodic variation in the Doppler frequency has been removed. The residual Doppler is mostly zero as we expect, but there is a trend in the azimuth direction which is likely a result of an inaccurate geometrical Doppler. By calculating the standard deviation of the estimated Doppler frequencies over several uncorrelated pixels from the uncorrected and the corrected Doppler grid, the corrected Doppler values showed a reduction of the standard deviation in the order of $0.5 \mathrm{~Hz}$, taking us very close to the model standard deviation of $2.5 \mathrm{~Hz}$.

\section{CONCLUSIONS}

We have presented a simple but effective way of correcting differences in gain at the raw data level assuming smooth raw data intensity variations in the azimuth direction. Our analysis shows that the correction reduces the variation in the estimated Doppler centroids which allows optimal performance of the high-precision Doppler estimation routine.

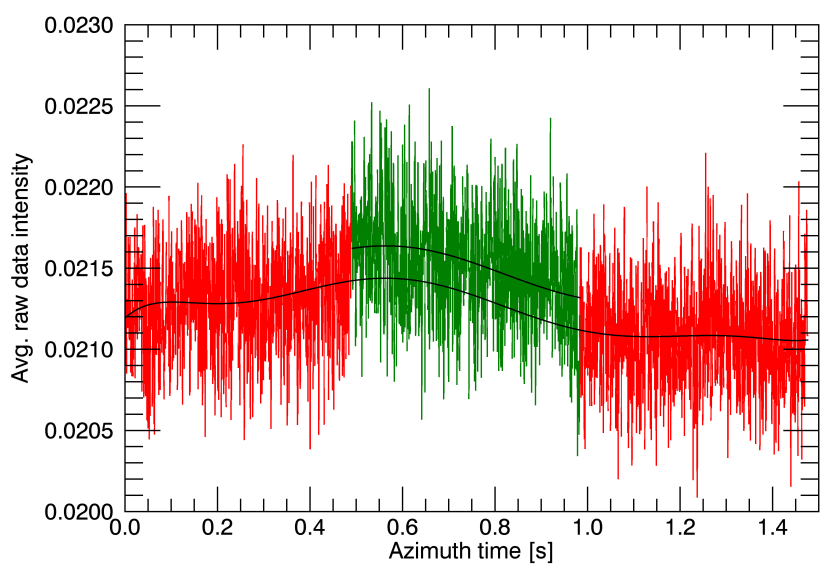

Fig. 3. Variation in gain between gates. The bottom line is a polynomial of order 7 fitted to the red data points. The top line is polynomial of same shape as the bottom one, but with a different offset fitted to the green data points.

\section{REFERENCES}

[1] Ian G. Cumming and Frank H. Wong, Digital processing of synthetic aperture radar data: algorithms and implementations, Artech House, 2005.

[2] Bertrand Chapron, Fabrice Collard, and Fabrice Ardhuin, "Direct measurements of ocean surface velocity from space: Interpretation and validation," Journal of Geophysical Research, vol. 110, no. C7, pp. 1-17, 2005.

[3] Alexis A. Mouche, Fabrice Collard, Bertrand Chapron, Knut-Frode Dagestad, Gilles Guitton, Johnny A. Johannessen, Vincent Kerbaol, and Morten Wergeland Hansen, "On the Use of Doppler Shift for Sea Surface Wind Retrieval From SAR," IEEE Transactions on Geoscience and Remote Sensing, vol. 50, no. 7, pp. 2901-2909, July 2012.

[4] Morten Wergeland Hansen, Kjell Kloster, Knut-Frode Dagestad, Stein Sandven, and Johnny A. Johannesen, "Retrieval of sea ice drift from SAR Doppler shift," in Proc. ESA Living Planet Symposium (SP-709), Bergen, 2010, pp. 1-5, ESA.

[5] Richard Bamler, "Doppler Frequency Estimation and the Cramér-Rao Bound," IEEE Transactions on Geoscience and Remote Sensing, vol. 29, no. 3, pp. 385-390, 1991.

[6] Geir Engen and Harald Johnsen, "High-precision Doppler frequency estimation for ocean applications," in Proc. SEASAR (SP-709), Troms $\emptyset, 2012$, pp. 1-4, ESA. 